\section{A simple confocal fibre-optic laser method for intraocular lens power measurement \\ IK Ilev}

\begin{abstract}
Purpose To develop novel confocal fibreoptic laser method (CFOLM) for accurate and objective measuring of the dioptric power of both positive and negative intraocular lenses (IOLs).
\end{abstract}

Methods The CFOLM principle of operation is based on a simple apertureless single-mode fibre laser confocal design. The key element is a single-mode fibre coupler that serves simultaneously as a point light source (3-5 $\mu \mathrm{m}$ fibre diameter) used for the formation of a collimated Gaussian beam, and as a confocal point receiver that is highly sensitive to spatial displacements of the focused backreflectance laser emission. The basic CFOLM systems include IOL testing set-ups for the measurement of both positive and negative IOLs.

Results The CFOLM designs provide high accuracy $(\leq 1 \mu \mathrm{m})$ in spatially locating the IOL focal point and in measuring the focal length in a broad range of both positive and negative powers including high-magnification IOLs with power greater than $\pm 20 \mathrm{D}$. We have tested various IOL samples with both positive $(+5$ to $+30 \mathrm{D})$ and negative $(-5$ to $-20 \mathrm{D})$ powers and we have obtained high levels of power testing repeatability estimated by a SD in the interval of $0.004-0.06 / 0.003-0.013 \mathrm{D}$ and a relative error in the interval of $0.015-0.3 / 0.02-$ $\mathbf{0 . 1 6 \%}$, for positive/negative IOLs, respectively. Conclusions The presented IOL power testing method offers a simple, accurate, objective, quick, and relatively inexpensive approach for dioptric power measurement of positive and negative IOLs. It provides an independent source of IOL power measurement data and information for evaluating the effectiveness and safety of novel IOL products.
Eye (2007) 21, 819-823; doi:10.1038/sj.eye.6702406; published online 28 July 2006

Keywords: intraocular lens dioptric power; confocal fibre-optic laser method; single-mode fibre coupler

\section{Introduction}

Since the invention and first implantation of intraocular lens (IOL) back in 1949, ${ }^{1}$ the development and use of IOLs has revolutionized refractive cataract surgery. An estimated 20.5 million Americans over the age of 40 years have cataracts in at least one eye and more than 2.9 million cataract surgeries are performed per year. ${ }^{2}$ The focal length (or dioptric power) is a fundamental parameter whose precise measurement is of critical importance for characterizing and evaluating the effectiveness and safety of a single focusing optical element or systems such as various positive and negative IOLs, contact lenses, eyeglasses, conventional lenses, objectives, and mirrors. Because of the complexity in accurately locating the principal focal planes that usually lie within the focusing element, various indirect methods for focal length measurement are conventionally used, such as image magnification, autocollimation, nodal slide, Bessel's method, Moiré deflectometry, and Talbot interferometry. ${ }^{3-9}$ The effectiveness of most of these methods is often limited with regard to high accuracy, dynamic range over which measurements can be performed (for both positive and negative dioptric powers), spatial sample alignment, and subjectively image observation. Recently, ${ }^{10-12}$ we have demonstrated a fibre-optic-based backreflectance technique for testing focusing optical elements with relatively large numerical
Division of Physics, Office of Science and Engineering Laboratories, Center for Devices and Radiological Health, US Food and Drug Administration, Rockville, MD, USA

Correspondence: IK llev, Division of Physics, Office of Science and Engineering Laboratories, Center for Devices and Radiological Health, US Food and Drug Administration, HFZ-130, 12725 Twinbrook Parkway, Rockville, MD 20857, USA

Tel: + 1301827 4685; Fax: + 13018274677

E-mail: ilko.ilev@fda.hhs.gov

Received: 14 December 2005

Accepted in revised form: 2 April 2006 Published online: 28 July 2006 
apertures and short positive focal lengths. This approach is compatible with high-resolution confocal laser microscopy and the combined fibre-optic confocal imaging systems offer advantages in terms of high spatial resolution, flexibility, miniaturization, and scanning potential. ${ }^{13,14}$ In this article, we demonstrate a novel and simple confocal fibre-optic laser method (CFOLM) for accurate, objective, and rapid measurement of a wide range of both positive and negative IOL dioptric powers.

\section{Methods}

The CFOLM operating principle and a basic optical design is illustrated in Figure 1a. ${ }^{13}$ The IOL dioptric power measurement method is based on an intensity sensing fibre-optic laser confocal approach. In this method, using a $2 \times 1$ single-mode fibre coupler, the output beam of an intensity-stabilized continuous wave laser is converted into a precisely collimated Gaussian laser beam that is focused on a backreflectance mirror surface by the focusing IOL $\left(L_{\text {test }}\right)$ to be tested. Because of the high sensitivity to spatial displacements of the focused backreflectance laser beam, the fibre-optic confocal arrangement provides precise location of the focal point when the total reflectance mirror is axially scanned and thus, a high accuracy in measuring the focal length of the test IOLs is achieved.

The key CFOLM idea involves the specific use of the single-mode fibre coupler that, because of its small core dimensions (3-5 $\mu \mathrm{m})$, performs simultaneously the following significant functions: (1) The single-mode fibre coupler provides effective launching and delivery of the input continuous wave low-power laser emission that might have various transverse-mode distribution and wavelength in the ultraviolet, visible, and infrared spectral ranges. (2) The output small-core-diameter single-mode fibre tip serves as a point confocal light

a

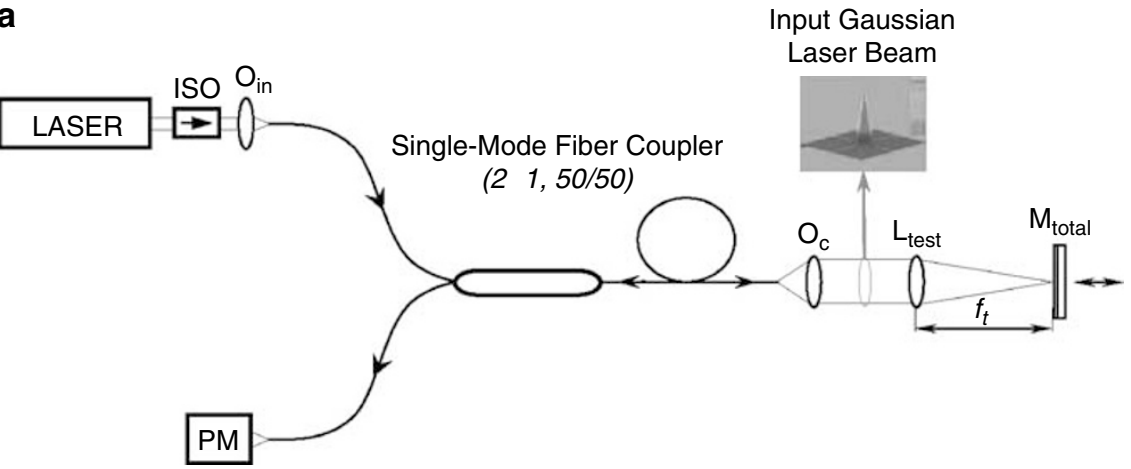

b

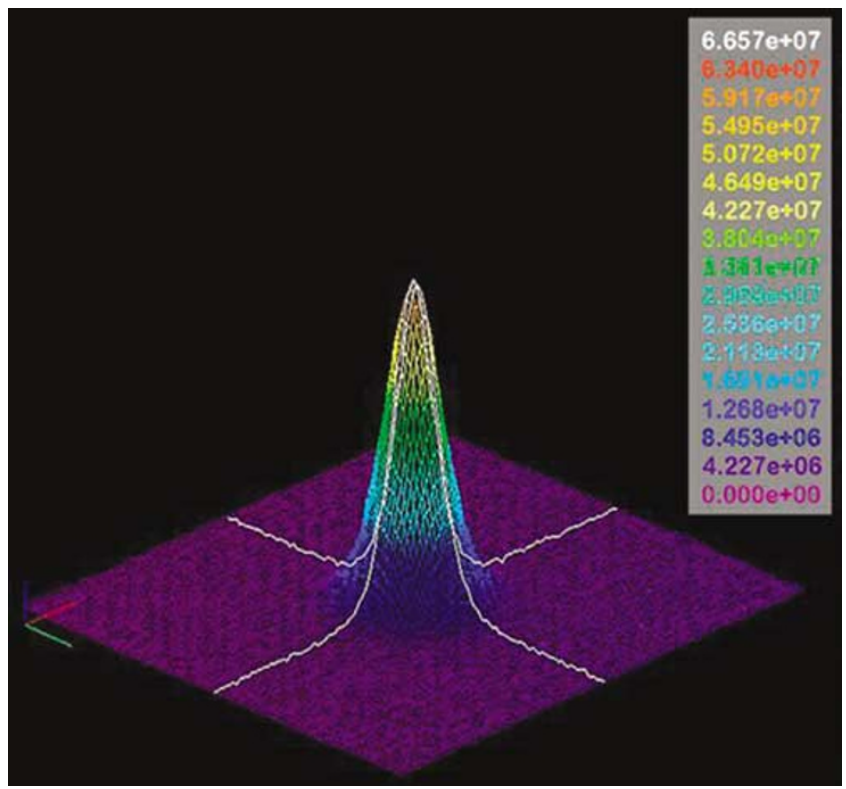

Figure 1 (a) Principal optical design of the CFOLM for IOL power measurement and (b) typical three-dimensional image of a Gaussian laser beam profile formed by the single-mode fibre coupler. 
source that ensures a Gaussian beam distribution with a typical profile shown in Figure 1b. In combination with the advantages gained by the use of a point-light-source fibre tip and an infinity-corrected objective $\left(Q_{c}\right)$, the Gaussian-mode distribution provides for precisely collimating the input beam directed onto the test focusing lens. (3) In comparison with the conventional pinhole-based confocal systems, which possess certain disadvantages related to significant signal attenuation, diffraction/aberration effects, misalignment problems, and inflexibility, the proposed focal-length test method is based on an apertureless fibre-optic confocal design. In this design, the same output single-mode fibre tip used as a point light source serves as a point receiver that is highly sensitive to spatial displacements of the focused backreflectance laser beam. (4) The fibre coupler provides delivery and intensity sensing of the spatially separated backreflected optical signals. The combination of these unique features of the proposed CFOLM design provides high accuracy (exceeding $1 \mu \mathrm{m}$ ) in spatially locating the focal point and therefore, in measuring the focal length of the test IOLs.

The CFOLM concept has no limitation with regard to numerical aperture and powers of the test focusing optics. An unique CFOLM feature is that it allows for the measurement of a wide range of both positive (converging) and negative (diverging) lenses, including high-magnification lenses and IOLs with power greater than $\pm 20 \mathrm{D}$. To get high accuracy and repeatability, the CFOLM approach requires some specific preliminary alignments such as laser-to-fibre coupling alignment providing maximum coupling efficiency; precise alignment of the system 'fibre-tip/collimating-objective', which produces a parallel collimated Gaussian laser beam; and IOL sample and backreflectance mirror alignment at normal incidence towards the collimated laser beam.

For positive IOL power measurements, the first step is to locate the focal point $F_{1}$ (see Figure $2 \mathrm{a}$ ) of the positive power IOL. The next step, using precise translational micrometric stages, is to measure the distance between the focal point $F_{1}$ and the lens's back vertex $V_{1}$. This distance is equal to the lens's back focal length $f_{\mathrm{b}}$ that is a fundamental parameter of practical importance. Then, using the directly measured $f_{\mathrm{b}}$, we can determine the effective focal length $f_{\text {eff }}$ of the IOL being tested. In case of either thin or planoconvex IOLs, the $f_{\text {eff }}$ is equal to the $f_{\mathrm{b}}$ (see Figure 2a). To determine the $f_{\text {eff }}$ for lenses with different shapes (for instance, a biconvex lens shown in Figure $2 b$ ), we can use the following dependence between $f_{\text {eff }}$ and $f_{\mathrm{b}}:^{3}$

$$
f_{\text {eff }}=f_{\mathrm{b}} /\left[1-t\left(n_{2}-n_{1}\right) / n_{2} r_{1}\right]
$$
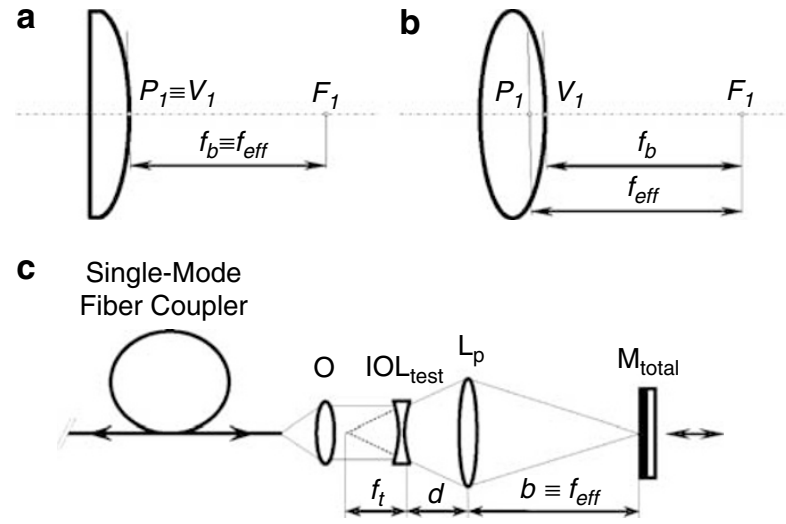

Figure 2 Location of the focal points $F_{1}$, principal points $P_{1}$, lens vertex points $V_{1}$, back focal lengths $f_{\mathrm{b}}$, and effective focal lengths $f_{\text {eff }}$ for a planoconvex (a) and a biconvex (b) positive power IOL. An additional set-up (c) to the principal optical design shown in Figure 1a for dioptric power measurement of negative power IOLs.

where $t$ is the lens thickness, $r_{1}$ is the front surface radius of curvature, and $n_{1}$ and $n_{2}$ are the refractive indexes of the lens and the surrounding medium (in air $n_{1}=1$ ), respectively. In case of thin $(t \approx 0)$ or planoconvex $\left(r_{1} \approx \propto\right)$ lenses, Equation (1) is reduced to the simple dependence $f_{\text {eff }}=f_{\mathrm{b}}$.

For negative IOL power measurements, the principal CFOLM design (Figure 1) is modified as shown in Figure 2c. It involves an additional conventional positive lens $L_{\mathrm{p}}$ (usually, a planoconvex lens) with known focal length $f_{\mathrm{p}}$. Then, using the classical lens equation $1 / f=1 /$ $a+1 / b$ for the negative/positive lens combination, we can get the following formula for determining the focal length $f_{\mathrm{t}}$ of the test negative lens $L_{\text {test }}$ :

$$
f_{\mathrm{t}}=f_{\mathrm{p}} b /\left(b-f_{\mathrm{p}}\right)-d
$$

where $d$ and $b$ are the distances between the two lenses, and the image to the positive lens, respectively. Thus, the CFOLM procedure for negative power IOL

measurements is reduced to spatial location of the focal point $F_{\mathrm{p}}$ and direct measurement of the distances $d$ and $b$.

\section{Results and discussions}

At present, the IOL dioptric power is conventionally determined in accordance with the International Standard, ISO 11979-2:1999 Ophthalmic

Implants - Intraocular Lenses, Part 2: Optical properties and test methods. ${ }^{9}$ This standard specifies the basic requirements that should be applied to the IOL dioptric power testing procedures including the allowed tolerances on IOL dioptric power such as $\pm 0.3 \mathrm{D}$ for the IOL dioptric power range of 0 to $\leq 15 \mathrm{D} ; \pm 0.4$ for $>15$ to $\leq 25 \mathrm{D} ; \pm 0.5$ for $>25$ to $\leq 30 \mathrm{D}$; and $\pm 1.0 \mathrm{D}$ for $>30 \mathrm{D}$. 
The standard describes also three reference methods for the determination of IOL dioptric power using calculation from measured dimensions, measured back focal length, and measured magnification. The applicability of these methods including the one that is widely utilized in the IOL industry back-focal length standard design is often limited by some of their specific deficiencies such as limited dynamic range over which measurements can be performed (ie at testing highpower positive IOLs); limited design potential for dioptric power testing of positive IOLs only; applicability limited to non-toric monofocal spherical IOLs only; limited accuracy and repeatability particularly at highpower IOL testing; complex sample spatial and beam collimation alignment; and subjective image observation. To improve some of these limiting features of the standard IOL power testing methods, especially when newly developed IOL products with higher quality and narrower dioptric power tolerance are tested, we have developed the alternative CFOLM approach described here that provides accurate and completely objective dioptric power testing of both positive and negative IOLs within an unlimited power range.

Using the proposed CFOLM designs illustrated in Figure 1a (for positive power IOLs) and Figure 2c (for negative power IOLs) as well as the CFOLM focal length measurement procedures described, we have tested various IOL samples with both positive (from +5 to $+30 \mathrm{D}$ ) and negative (from -5 to $-20 \mathrm{D}$ ) dioptric powers. These power ranges are among the most typically used, in practice, IOL dioptric powers. However, there are no principal limitations for the suggested CFOLM designs to be exploited for testing of various positive/negative IOLs having dioptric powers beyond these ranges including the power interval of -5 to $+5 \mathrm{D}$. In this case, special precautions for the mechanical stability of the testing CFOLM system should be taken, such as the use of vibration-isolated optical tables and long-range micrometric stages. Moreover, because the CFOLM testing systems have no limitation also with regard to the wavelength range of the laser source used (the only requirement is related to the optical fibre coupler that should be a single-mode one within the laser wavelength range), we have utilized laser sources with various wavelengths in the green/red visible spectral range including a $\mathrm{He}-\mathrm{Ne}$ laser with a wavelength of $543.5 \mathrm{~nm}$, which is within the spectral interval of $546 \pm 10 \mathrm{~nm}$ required from the ISO standard. We have measured the IOL powers under two environmental conditions: (1) in air with dry IOL samples, and (2) in an in situ simulation using glass or quartz cuvettes with IOL samples in balanced salt solutions of various concentrations. In order to test the CFOLM repeatability of IOL power measurement, each positive and negative power IOL has been tested at least three times at identical environmental conditions. For the positive IOL dioptric power measurements, we have obtained high repeatability with an estimated standard deviation in the range of $0.004-0.06 \mathrm{D}$ and a relative error in the range of $0.015-0.3 \%$. For the negative IOL dioptric power measurements, the test repeatability estimated by the standard deviation and relative error was in the interval of $0.003-0.013 \mathrm{D}$ and $0.02-0.16 \%$, respectively. Thus, in addition to the CFOLM advanced features described (such as the potential for testing of positive/ negative, low-/high-power and monofocal/multifocal IOLs using simple, precise, and objective alignment procedures), these values demonstrate the CFOLM potential for significantly improved accuracy $(<1 \mu \mathrm{m}$ in locating the IOL focal point vs more than several tens of microns for the standard test methods ${ }^{8,9}$ ) and repeatability (typically $<0.03 \mathrm{D}$ vs $0.15 \mathrm{D}$ using the standard test methods ${ }^{8,9}$ ) in IOL power testing that is an essential advantage when newly developed $\mathrm{IOl}$ products should be tested.

The CFOLM accuracy of IOL power measurement depends on the following basic factors. First, the accuracy of focal point location depends upon the mirror displacement accuracy and the laser power/detection system signal fluctuations. Using an intensity-stabilized laser $(<0.1 \%$ power stability), a sensitive photodetecting system, and a $1 \mu \mathrm{m}$ linear mirror displacement, the signal levels registered at the maximum of the confocal response curve are higher than the signal fluctuations, which do not exceed $1 \%$. Thus, an accuracy of $1 \mu \mathrm{m}$ (or possibly $<1 \mu \mathrm{m}$ at lower signal fluctuations) in focal point location and therefore, of focal length measurement is achieved. Second, the use of a single-mode fibre coupler ensures a Gaussian-mode distribution utilized for precise collimating and focusing of the input and backreflectance beams. However, this distribution also requires a correction $\left[\pi^{2} \omega_{0}^{4} / \lambda^{2}(a-f)\right]\left(\omega_{0}\right.$ is the characteristic Gaussian-mode beam radius) to the distance $a$ in the lens equation. ${ }^{15}$ In our case this addition is negligible owing to the small value of the single-mode core radius. Third, the influence of the aberration effects on the CFOLM measurement accuracy is negligible because a monochromatic laser emission is used, the mirror displacement is along the axis with additional angular adjustment, and the laser beam distribution is Gaussian with strongly decreasing intensity in the laser spot periphery. Moreover, in comparison with the conventional IOL power testing methods where it is quite challenging to get a highly collimated light/laser beam, the CFOLM designs are based on the use of a few micron core-diameter single-mode fibre as point light source and point receiver as well as infinity-corrected focusing optics, which provides near-to-the-ideal 
conditions for the formation and collimation of a Gaussian laser beam with various beam diameters. This advanced CFOLM feature ensures a highly accurate IOL power measurement related to the IOL paraxial focus.

\section{Conclusion}

We have demonstrated a simple CFOLM for measuring the dioptric power of various IOLs. This method features the following advantages. (1) The single-mode fibre coupler serves simultaneously as a point light source and a sensitive confocal point receiver that provides near-tothe-ideal conditions for forming and receiving the Gaussian beam. (2) The CFOLM designs provide high accuracy $(\leq 1 \mu \mathrm{m})$ in focal point locating and in measuring the focal length of various positive and negative IOLs. (3) The CFOLM operating principle and CFOLM designs provide a simple, accurate, completely objective, quick, and relatively inexpensive method for measuring the focal length of various focusing optical elements and systems including IOLs, contact lenses, eyeglasses, conventional lenses, objectives, and mirrors.

\section{Acknowledgements}

I thank Don Calogero, Robert Faaland, and Robert Landry for their constant support and stimulated discussions on this work.

\section{References}

1 Apple D, Sims J. Harold Ridley and the invention of the intraocular lens. J Surv Ophthalmol 1996; 40: 279-292.
2 National Eye Institute. Arch Ophthalmol, (accessed June 22, 2005),http://www.nei.nih.gov/eyedata/pbd6.asp.

3 Smith WJ. Modern Optical Engineering, 2nd ed. McGraw-Hill: New York, 1990.

4 Nakano Y, Murata K. Talbot interferometry for measuring the focal length of a lens. Appl Opt 1985; 24: 3162-3166.

5 Su D, Chang C. A new technique for measuring the effective focal length of a thick lens or a compound lens. Opt Commun 1990; 78: 118-122.

6 Keren E, Kreske K, Kafri O. Universal method for determining the focal length of optical systems by moire deflectometry. Appl Opt 1988; 27: 1383-1385.

7 Tognetto D, Sanguinetti G, Sirotti P, Cecchini P, Marcucci L, Ballone $\mathrm{E}$ et al. Analysis of the optical quality of intraocular lenses. Invest Ophthalmol Vis Sci 2004; 45: 2682-2690.

8 Norrby N, Grossman L, Geraghry E, Kreiner C, Mihori M, Parel A et al. Accuracy in determining intraocular lens dioptic power assessed by interlaboratory tests. J Cataract Refract Surg 1996; 22: 983-987.

9 International Standard. Ophthalmic Implants - Intraocular Lenses_-Part 2: Optical Properties and Test Methods, ISO 11979-2 1999.

10 Ilev I. Simple fiber-optic autocollimation method for determining the focal lengths of optical elements. Opt Lett 1995; 20: 527-529.

11 Ilev I, Uttamchandani D, Culshaw B. Fiber-optic backreflectance method for determining the effective focal lengths of optical elements. Appl Opt 1996; 35: 716-718.

12 Ilev I, Kumagai H, Toyoda K, Uttamchandani D, Culshaw B. Alternative fiber-optic backreflectance sensor for simultaneously measurement of the focal lengths of optical elements and distances. Opt Rev 1997; 4: 58-60.

13 Ilev I. Pending Patent No. 60/668,239, March 3, 2005.

14 Flusberg BA, Cocker ED, Piyawattanametha W, Jung JC, Cheung EL, Schnitzer MJ. Fiber-optic fluorescence imaging. Nat Methods 2005; 2: 941-950.

15 Self S. Focusing of spherical Gaussian beams. Appl Opt 1983; 22: 658-661. 